\title{
Polymorph of $N, N^{\prime}$-di-n-butylperylene-3,4:9,10-bis(dicarboximide) and their electronic structure
}

\author{
Jin Mizuguchi \\ Department of Applied Physics, Graduate School of Engineering \\ Yokohama National University \\ 240-8501 Yokohama, Japan
}

Perylene pigments are known to exhibit a variety of shades in the solid state from vivid red, via maroon to black, although their solution spectra are quite similar. A second phase of the title compound (phase II) has newly been found when the single crystals were grown from the vapor phase. Phase II bears a black color while the previous phase (phase I) exhibits red-maroon. For this reason, the electronic structure of both phases has been investigated on the basis of the crystal structure as well as intermolecular interactions. In both phases, the absorption spectrum is composed of two bands: one is due to individual molecules (i.e. around 450-550 nm) and the second band arises from excitonic interactions between transition dipoles (i.e. around $600 \mathrm{~nm}$ in phase I and 625 $\mathrm{nm}$ in phase II). The intermolecular interactions along the stacking axis are primarily responsible for the appearance of the second band in both phases. Additionally, the interaction on the molecular plane is found to be slightly hypsochromic in phase I while bathochromic in phase II. This induces a difference in absorption maximum of the second band, leading to two different colors (red-maroon and black) in phases I and II.

Keywords: perylene pigment; electronic structure; crystal structure; polymorph 


\section{Introduction}

Perylene derivatives are well-known organic pigments which exhibit a variety of shades in the solid state from vivid red, via maroon to black, although no significant difference is recognized in solution spectra [1]. These compounds have attracted attention as colorants [1], electrophotographic photoreceptors [2,3], photovoltaic elements [4] and optical disks [5]. Because the electronic characterization is most basic to an understanding of the color in the solid state as well as to electronic applications, we have so far carried out a series of investigations on the color generation mechanism of $\begin{array}{llll}\text { representative } & \text { perylene } & \text { (PR149 }\end{array}$ $N, N$ '-bis(3,5-xylyl)perylene-3,4:9,10-bis(dicarboximide), $\quad$ PR179 (maroon: $N, N^{\prime}$-dimethylperylene-3,4:9,10-bis(dicarboximide) $\quad$ and PB31 (black: $N, N$ '-bis(2-phenylethyl)perylene-3,4:9,10-bis(dicarboxyimide)) on the basis of the crystal structure [6-9] and intermolecular interactions [5,10]. Our conclusion can be summarized as follows. The solid-state color of perylene pigments is mainly determined by two absorption bands in the visible region. The shorter-wavelength band is of molecular character that appears in common in all perylene derivatives while the longer-wavelength band is caused by resonance interactions between transition dipoles. Therefore, the color in the solid state can variously change, depending on the extent of resonance interactions based on the molecular arrangement. The red color appears when the interaction is quite small; whereas the maroon and black colors are characterized by medium and strong interactions, respectively [10].

On the otherhand, Hädicke and Graser have published in 1986 a number of crystal structures of perylene pigments which also include the structure of the title compound ( $N, N^{\prime}$-di-n-butylperylene-3,4:9,10-bis(dicarboximide) abbreviated to BP; Fig.1) [9,10]. 
In the meantime, the second phase of PB31 has been found by us [8] and characterized as described above [5]. Quite recently, the second phase of BP has also been isolated when grown from the vapor phase [11]. Interesting to say, phase II is black while the previous phase as obtained by recrystallization from nitrobenzene exhibits red-maroon. For this reason, electronic characterization of both phases has been conducted on the basis of the crystal structure and intermolecular interactions.

\section{Experiment}

\subsection{Materials and crystal growth of single crystals}

BP was synthesized by reaction of perylene tetracarboxylic dianhydride with n-butylamine in imidazol at $433 \mathrm{~K}$ for one hour in an autoclave. The product was purified by sublimation under argon at about $673 \mathrm{~K}$, using a two-zone furnace [12]. Single crystals of phase I were recrystallized from solution in nitrobenzene: whereas the single crystals of phase II were grown from the vapor phase in a closed system at about $680 \mathrm{~K}$. Both single crystals were used for structure analysis as well as for measurements of polarized reflection spectra.

Evaporated thin films of BP were prepared onto plain glass slides (film thickness: about $1000 \AA ̊$ ) using conventional vacuum equipment (Tokyo Vacuum Co. Ltd.: model EG240). The evaporated films were exposed to acetone vapor for one hour to bring about spectral changes. Acetone vapor loosens the crystal lattice, thus allowing the molecules to slide and/or rotate to find energetically more stable sites. 


\subsection{Measurements}

UV-Vis spectra were recorded on a UV-2400PC spectrophotometer (Shimadzu). The temperature dependence of the absorption spectra in evaporated films were measured in the range between 20 and $300 \mathrm{~K}$ on a UV-2400PC spectrophotometer in combination with a cryostat from Iwatani Gas Co. Ltd. (model: CRT-105-OP). Diffuse reflectance spectra were measured on powders on a UV-2400PC spectrophotometer together with an integrating sphere attachment (ISR-240A from Shimadzu). Measurements for polarized reflection spectra were made on single crystals by means of a UMSP80 microscope-spectrophotometer (Carl Zeiss). An Epiplan Pol $(\times 8)$ objective was used together with a Nicol-type polarizer. Reflectivities were corrected relative to the reflection standard of silicon carbide.

\subsection{Molecular Orbital (MO) Calculations}

The geometry of the molecule was optimized by means of AM1 Hamiltonian of MOPAC program package [13]. The INDO/S program used for spectroscopic calculations is part of the ZINDO program package [14]. The spectroscopic calculations were made for the optimized geometry as well as for the molecule extracted from the crystal lattice of phases I and II using the X-ray coordinates.

\section{Results and Discussion}

\subsection{Crystal structure of phases I and II}


Table 1 summarizes the crystallographic parameters of phases I [6] and II [11]. In both modifications, the crystal system and molecular symmetry are monoclinic and $C_{\mathrm{i}}$, respectively. The space groups are $P 21 / c$ and $P 21 / n$ for phases I and II, respectively. The unit cell of phase II is approximately twice as big as that of phase I. Figs.2(a) and 2(b) show the molecular arrangement of phase I on the $(b, c)$ plane and along the molecular stack, respectively. On the molecular plane, the molecules are not oriented parallel, but arranged in a zigzag fashion along the $b$-axis. It is interesting to note that the alkyl chains connected to the imide site are not extended along the long molecular axis, but are standing on the molecular plane (Fig.2(b)). The slip angle and the interplanar distance for the molecular stack are about $48^{\circ}$ and $3.6 \AA$, respectively.

In phase II, there are two independent molecules (A \& B) in the unit cell and both molecules are on different centro-symmetric sites. Each conformation is, however, slightly different in geometry of the alkyl chains as described below. Figs.3(a) and 3(b) show the molecular arrangement of phase II on the $(a, c)$ molecular plane and along the stacking $b$-axis. On the molecular plane (Fig.3(a)), molecules A and B are arranged alternately along the $a$ and $c$-axes. Each molecules are also aligned alternately in a fashion "upward \& downward" along the $(a, c)$-diagonal direction. Molecules A and B are stacked independently side by side along the $b$-axis. In molecule $\mathrm{A}$, as shown in Fig.3(b), the butyl chains are extended in a zigzag fashion along the long molecular axis up to the third $\mathrm{C}$ atom and then the fourth $\mathrm{C}$ atom of the methyl group is bent almost parallel to the molecular plane. On the other hand, in molecule $\mathrm{B}$, the alkyl chains are standing on the molecular skeleton in a similar fashion to that of phase I (Fig.2(b)). The slip angle and the interplanar distance for A-A and B-B pairs are found to be nearly the same: about $48^{\circ}$ and $3.5 \AA$, respectively. 
3.2 MO calculations and solution spectra

Geometry optimization of MO calculations gave a heat of formation of $144.8 \mathrm{kcal} / \mathrm{mole}$. The spectroscopic calculations revealed only one electronic transition for the optimized geometry, as well as for the molecule in phase I and molecules A \& B in phase II. This is assigned to the HOMO/LUMO $\pi-\pi^{*}$ transition. The absorption maximum and oscillator strength are: $435.3 \mathrm{~nm}$ and 1.286 for the optimized geometry. For the molecules extracted from the crystal lattice: $450.7 \mathrm{~nm}$ and 1.32 for phase I, and 438.9 $\mathrm{nm}$ and 1.26 for molecule A in phase II while $444.7 \mathrm{~nm}$ and 1.28 for molecule $\mathrm{B}$ in phase II. The direction of the transition dipole points in all molecules along the long molecular axis as shown in Fig.1.

\subsection{Solution spectrum and solid-state spectrum in evaporated films}

Fig.4 shows the solution spectrum of $\mathrm{BP}$ in dimethylsulfoxide and the solid-state spectrum of evaporated BP. The solution spectrum exhibits a typical progression of absorption bands starting from $525 \mathrm{~nm}$ with a spacing about $1400 \mathrm{~cm}^{-1}$. MO calculations showed that there is only one $\pi-\pi^{*}$ electronic transition in the visible region, so that the longest-wavelength band is attributed to the pure electronic band as designated by $0-0$, followed by the $0-1,0-2$ and $0-3$ vibronic transitions as shown. In the solid-state spectrum of evaporated BP (whose phase is amorphous as shown below), the absorption maxima are slightly displaced toward longer wavelengths, accompanied by different absorption intensities. Nevertheless, one-to-one correspondence of all 
absorption bands between the solution and solid-state spectra is possible, indicating that the molecular nature is well preserved even in the solid state.

3.4 Changes in absorption spectra and X-ray diffraction diagrams due to vapor treatment

Figs.5(a) and 5(b) show the absorption spectra and X-ray diffraction diagrams before and after vapor treatment. Vapor treatment brings about an additional band at longer-wavelengths (about $580 \mathrm{~nm}$ ) and this new band is evidently attributed to intermolecular interactions because it appears on going from solution to the solid state.

The broad band around $2 \theta=27^{\circ}$ in Fig.5(b) is due to the glass substrate used. The X-ray diffraction diagram before vapor treatment gives a halo pattern, indicating that the evaporated film is amorphous. Then, vapor treatment induces crystallization as shown by diffraction peaks around $2 \theta=6,11,19$ and $25^{\circ}$. These peaks are mostly assigned to phase I as shown. It is now evident that the new band appears as a result of the phase change from an amorphous to the crystalline state. The origin of the new band will be discussed later in Section 3.7.

It is also to be noted that the above spectral and crystallographic changes were also observed for heat treatment at $100{ }^{\circ} \mathrm{C}$ for 30 minutes.

\subsection{Temperature dependence of absorption spectra in evaporated films}

Since the new band around $580 \mathrm{~nm}$ arises from intermolecular interactions, the present band is supposed to exhibit a significant temperature dependence at low 
temperatures due to enhanced intermolecular interactions caused by lattice contraction. Fig.6 shows the spectral changes of evaporated BP after vapor treatment as a function of temperature. The longest-wavelength band exhibits an appreciable temperature dependence and is also displaced toward longer wavelengths, accompanied by several isosbestic points. On the other hand, the shorter-wavelength bands due to individual molecules remain relatively unaffected. The present temperature dependence evidently bears out that the longer-wavelength band is due to intermolecular interactions.

3.6 Polarized reflection spectra measured on single crystals and resonance interactions between transition dipoles

Fig.7 shows the polarized reflection spectra measured on the $(a, b)$ plane of single crystals of phase I together with the corresponding projection. A prominent reflection band appears around $600 \mathrm{~nm}$ together with smaller bands around 480 and $530 \mathrm{~nm}$ for polarization perpendicular to the $a$-axis. The present spectrum is in good agreement with the absorption spectrum of evaporated BP after vapor treatment, especially at $20 \mathrm{~K}$ (Fig.6). Polarization parallel to the $a$-axis gives a similar but less intensified spectrum. This result is well understood in terms of the direction of the transition dipole of the molecule as shown in Fig.1. As shown in 7(b), the projection of the vector component of the transition dipole is larger onto the $b$-axis (i.e. perpendicular to the $a$-axis) than onto the $a$-axis,. This leads to a higher reflection for polarization perpendicular to the $a$-axis than parallel to it.

Likewise, phase II gives similar reflection bands composed of two bands in the visible region as shown in Fig.8(a). Polarization measurements were made on the $(a, b)$ 
plane, on which the molecules are arranged along the $a$ (or $b$ ) axis with an angle of $45^{\circ}$ (Fig.8(b). Therefore, the projection of the transition dipole onto the $a$-axis gives nearly the same contribution to the reflection intensity as that of the $b$-axis as shown in Fig.8(a). Since the two absorption bands cover nearly the whole visible region, the color appears almost black.

\subsection{Assignment of the long-wavelength band}

We have pointed out in our previous reports $[5,10,15,16]$ that, in organic pigments, the excitonic interactions play an important role in the determination of electronic structure (i.e. color) in the solid state. There are two requirements for the occurrence of excitonic interactions in the solid state. (a) the component molecules possess a large transition moment and (b) the molecules are arranged in a periodic array [17].

When an excitation induces a transition dipole in the molecule, the excited state in crystals involves wavefunctions with significant probabilities on nearest neighbors. Therefore, the exciton coupling may well involve energy contributions from interactions with all of these nearest neighbor molecules acting in concert in the lattice. This may lead to a band splitting called Davydov splitting of the excited state or spectral displacement toward longer or shorter wavelengths. The interaction energy ( $\left.\Delta E_{\text {exciton }}\right)$ is given by the dipole-dipole equation [17]: $\Delta E_{\text {exciton }}=\left|\mu_{T}\right|^{2}\left(1-3 \cos ^{2} \theta\right) / r^{3}$, where the transition dipole is denoted by $\mu_{\mathrm{T}}$, the distance and angle between two transition dipoles by $r$ and $\theta$, respectively. As evident from the present equation, the overall spread or shift energy is determined by the strength of the interneighbor coupling $\left(\left|\mu_{\mathrm{T}}\right|^{2}\right)$ which is directly proportional to the absorption coefficient of the molecule as well as by the 
mutual relative orientation of the transition dipoles in molecular assemblies. That is, the term $\left(1-3 \cos ^{2} \theta\right) / r^{3}$ determines the geometrical relationship of transition dipoles correlated with the crystal structure. The bathochromic or hypsochromic shift depends on the critical angle of $\theta=54.7^{\circ}$, below which the former will result ("head-to-tail"-like arrangement) and above which the latter will be the case ("parallel”-like arrangement). The present excitonic interaction is especially significant in pigment systems because their absorption coefficients are quite large. In fact, the molar absorption coefficient of BP is as large as about 20,000 as shown in Fig.4.

Let us consider the excitonic interaction in phases I and II on the molecular plane as well as along the stacking axis. It is important to bear in mind the geometrical factor $\left(\left(1-3 \cos ^{2} \theta\right) / r^{3}\right)$ and the direction of the transition dipole $\left(\mu_{T}:\right.$ Fig.1). In Fig.2(a), the translationally-equivalent molecules lie at the four corner of the unit cell. The interaction between transition dipoles along the $c$-axis (distance: $9.40 \AA$ ) is larger than along the $b$-axis (distance: $28.23 \AA$ ) by a factor of about 27 . The slip angle between two molecules is about $58^{\circ}$. Therefore, this pair makes a hypsochromic contribution. On the other hand, the translationally-inequivalent pair between molecules at the corner and center of the unit cell is supposed to cause a Davydov splitting to occur. However, this interaction is about one-fourth of the one caused by molecule pairs along the $c$-axis as judged from the geometrical factor. Now, we look at the stack pair shown in Fig.2(b). The interplanar distance amounts to about $3.6 \AA$ and the slip angle is about $48^{\circ}$. The geometrical term of the stack pair is, by far, larger than that of the in-plane pair along the $c$-axis by a factor of about 18 . Furthermore, the slip angle $\left(48^{\circ}\right)$ is below the critical angle of $54.7^{\circ}$. So the stack pair contributes significantly to the bathochromic shift. The present bathochromically-displaced band corresponds to the one around $600 \mathrm{~nm}$ in 
Fig.7(a).

In phase II, the translational-equivalent molecules B are located at the four corners of the unit cell as shown in Fig.3(a). However, molecule B at the center is traslationally-inequivalent. That is, molecules B are arranged alternately in a fashion "upward \& downward” along the $(a, c)$-diagonal direction. So the excitonic interaction between translationally-equivalent A-A or B-B molecules along the $a$-axis (slip angle: $53.5^{\circ}$; distance: $18.41 \AA$ ) is considered quite small as judged from the distance $r$ in the geometrical term $\left.\left(1-3 \cos ^{2} \theta\right) / r^{3}\right)$. For the same reason, the contribution due to A-A or B-B molecules along the $c$-axis is considered even smaller. On the other hand, both A-A \& B-B stack pairs as shown in Fig.3(b) contribute considerably to the bathochromic displacement, because the slip angle $\left(48^{\circ}\right)$ is below the critical angle of $54.7^{\circ}$ and the interplanar distance is about $3.5 \AA$.

Judging from the excitonic interactions described above, it is obvious that the stack pair governs primarily the bathochromic shift in phases I and II. The extent of this interaction is, more or less, of the same order in both phases. However, the interaction on the molecular plane plays an additional role. This interaction is slightly hypsochromic in phase I while bathochromic in phase II. (phase I: $58^{\circ}$; phase II: $53.5^{\circ}$ ). In total, the extent of the bathochromic shift is larger in phase II than in phase I. This interprets why the second band due to excitonic interactions appears at slightly longer wavelengths in phase II than in phase I (Figs. 7 and 8).

\section{Conclusions}

The electronic structure of phases I and II of BP has been investigated on the basis of 
the crystal structure as well as intermolecular interactions. In both phases, the absorption spectrum is composed of two bands: one is due to individual molecules around 450-550 nm and the second band arises from excitonic interactions between transition dipoles (around 600-650 nm). The excitonic interactions along the stacking axis are primarily responsible for the appearance of the second band. Secondarily, the interaction on the molecular plane induces an additional contribution to the spectral shift. In total, the second band appears at longer wavelengths in phase II than in phase I. 


\section{References}

[1] Herbst, M; Hunger, K. Industrial Organic Pigments: VCH; 1993: 467.

[2] Borsenberger, P M; Regan, M T; Staudenmayer, W. J.US patent 4,578,334: 1984.

[3] Gregg, B. A. J. Phys. Chem. 1996; 100: 852.

[4] Mizuta, Y; Miyamoto, E; Azuma, J; Nakazawa, T. Proc. of Japan Hardcopy’04; 2004: 175.

[5] Mizuguchi J. J. Appl. Phys. 1998; 84: 4479.

[6] Hädicke, E; Graser, F. Acta Crystallogr. 1986; C42: 189.

[7] Hädicke, E; Graser, F. Acta Crystallogr. 1986; C42: 195.

[8] Mizuguchi, J. Acta Cryst. 1998; C 54: 1479.

[9] Mizuguchi, J; Tojo, K. Z. Krist. NCS 2001; 216: 375.

[10] Mizuguchi, J; Tojo, K. J. Phys. Chem. 2002; B106: 767.

[11] Mizuguchi, J. Z. Krist. NCS. 2003; 218: 131.

[12] Mizuguchi, J. Krist. Tech. 1981; 16: 695.

[13] WinMOPAC Ver.3, Fujitsu.

[14] Zerner, M. C. ZINDO, A General Semi-Empirical Program Package. Department of Chemistry, University of Florida, Gainesville, FL.

[15] Endo, A; Matsumoto, S. J. Mizuguchi, J. Phys. Chem. A, 1999; 103: 8193.

[16] Mizuguchi, J. J. Phys. Chem. A, 2000; 104: 1817.

[17] Kasha, M. Spectroscopy of the Excited State, Plenum Press; 1976: 337. 
Figure captions:

Fig. 1 Molecular conformation of BP. The dotted line denotes the direction of the transition dipole $\left(\mu_{\mathrm{T}}\right)$ as deduced from MO calculations.

Fig. 2 (a) Projection of the crystal structure of phase I onto the (b,c) plane and (b) molecular stack for two molecules.

Fig. 3 (a) Projection of the crystal structure of phase II onto the $(a, c)$ plane and (b) molecular stack for A-A and B-B pairs.

Fig. 4 Solution spectrum of BP in dimethylsulfoxide and solid-state spectrum of evaporated BP.

Fig. 5 Absorption spectra and X-ray diffraction diagrams of evaporated BP before and after vapor treatment.

Fig. 6 Temperature dependence of evaporated BP after vapor treatment measured in the temperature range between 20 and $300 \mathrm{~K}$.

Fig. 7 (a) Polarized reflection spectra measured on the $(a, b)$ plane of single crystals of phase I and (b) projection of the crystal structure onto the $(a, b)$ plane.

Fig. 8 (a) Polarized reflection spectra measured on the $(a, b)$ plane of single crystals of phase II and (b) projection of the crystal structure onto the $(a, b)$ plane. 
Table 1 Crystallographic parameters for phases I and II.

\begin{tabular}{ccc}
\hline & phase I [6] & phase II [11] \\
\hline Formula & $\mathrm{C}_{32} \mathrm{H}_{26} \mathrm{~N}_{2} \mathrm{O}_{4}$ & $\mathrm{C}_{32} \mathrm{H}_{26} \mathrm{~N}_{2} \mathrm{O}_{4}$ \\
Crystal system & monoclinic & monoclinic \\
Space group & $P 21 / c$ & $P 21 / n$ \\
Molecular symmetry & $C_{\mathrm{i}}$ & $C_{\mathrm{i}}$ \\
$Z$ & 2 & 4 \\
$a(\AA)$ & $4.734(1)$ & $18.41(1)$ \\
$b(\AA)$ & $28.233(4)$ & $4.630(3)$ \\
$c(\AA)$ & $9.396(1)$ & $27.61(2)$ \\
$\beta\left({ }^{\circ}\right)$ & $110.86(1)$ & $105.74(5)$ \\
\hline
\end{tabular}

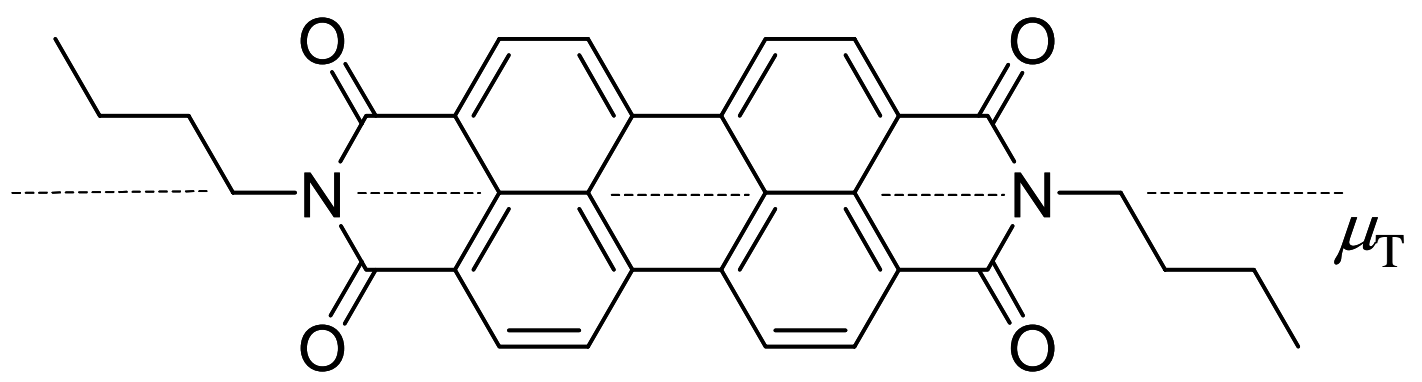

Fig.1 
(a)

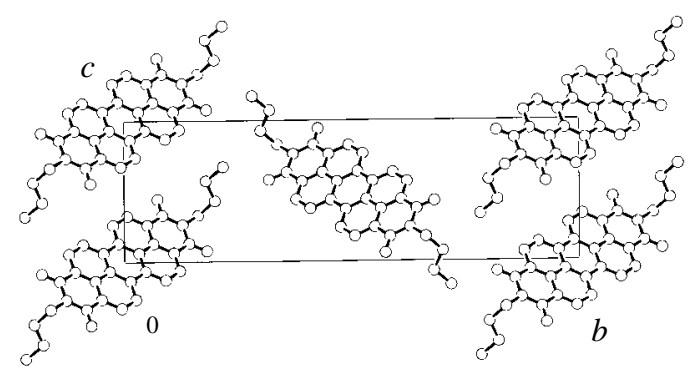

(b)

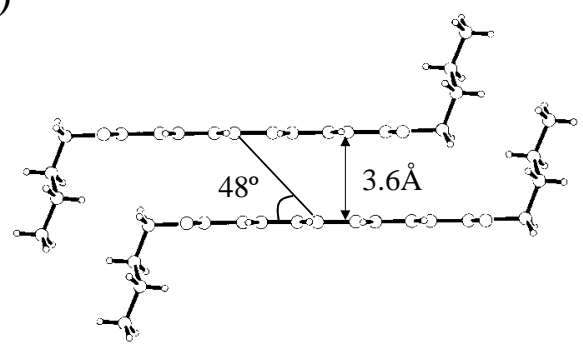

(a)

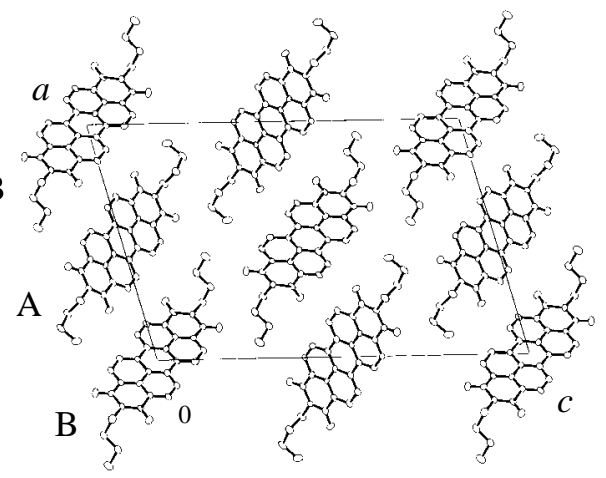

(b)

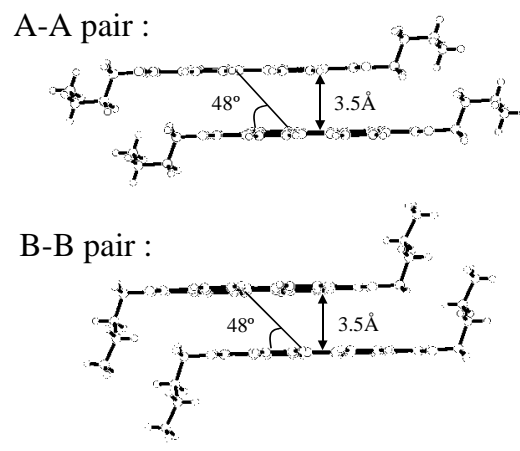

Fig.3

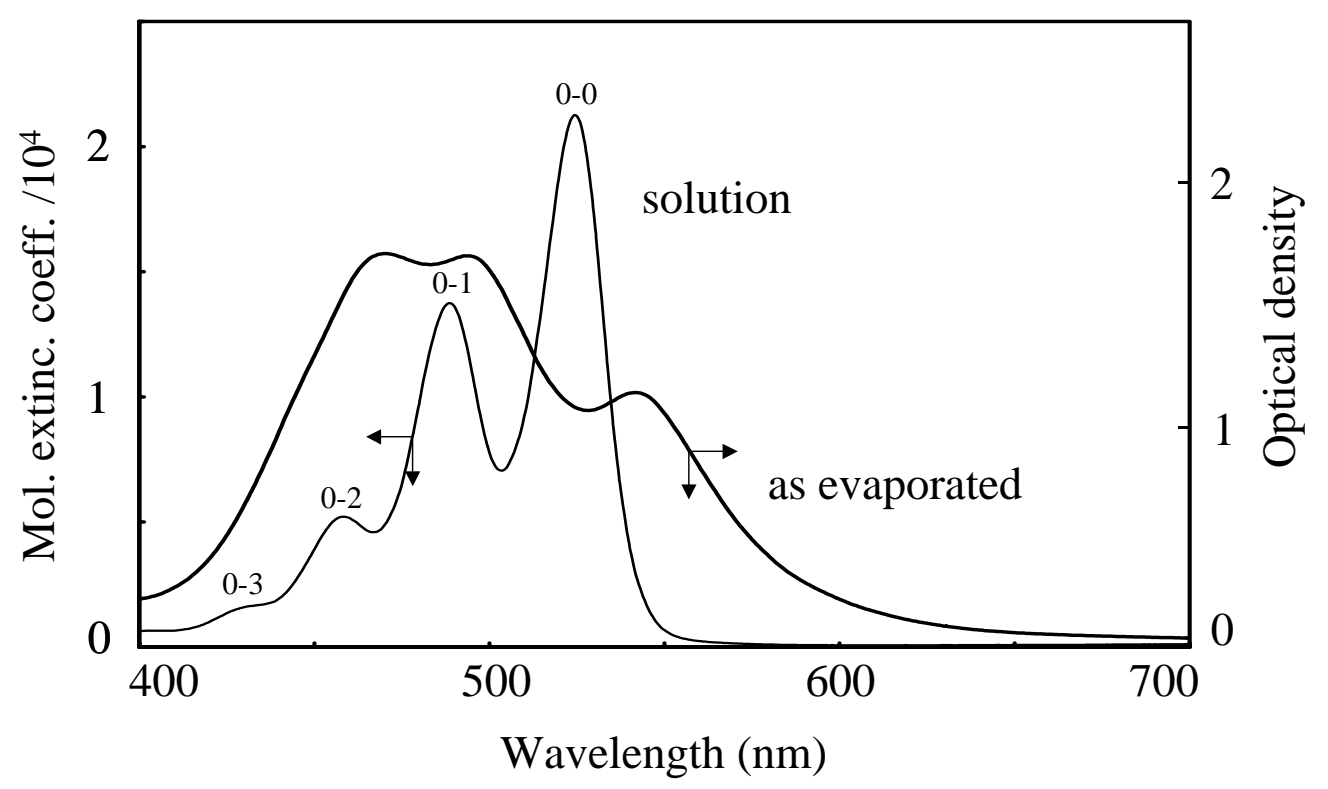

Fig. 4 
(a)

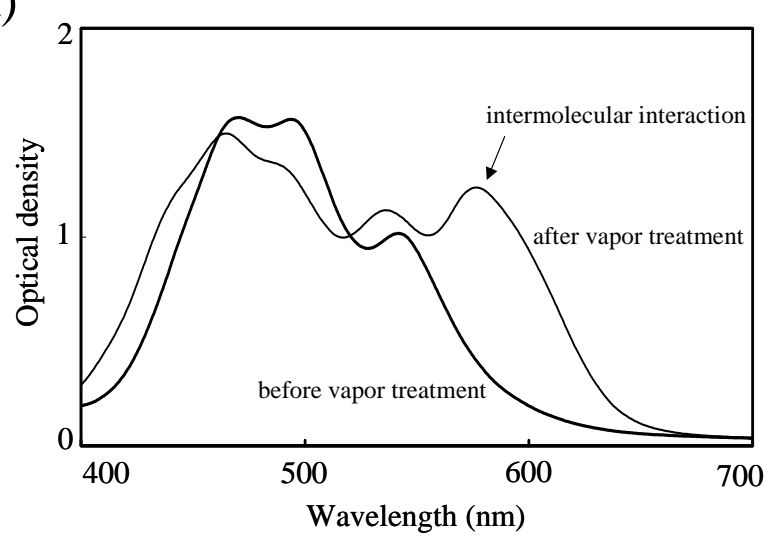

(b)

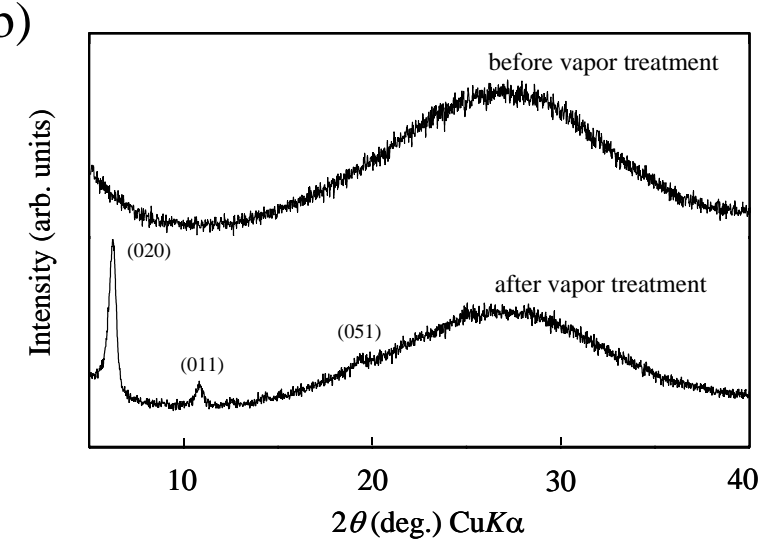

Fig. 5

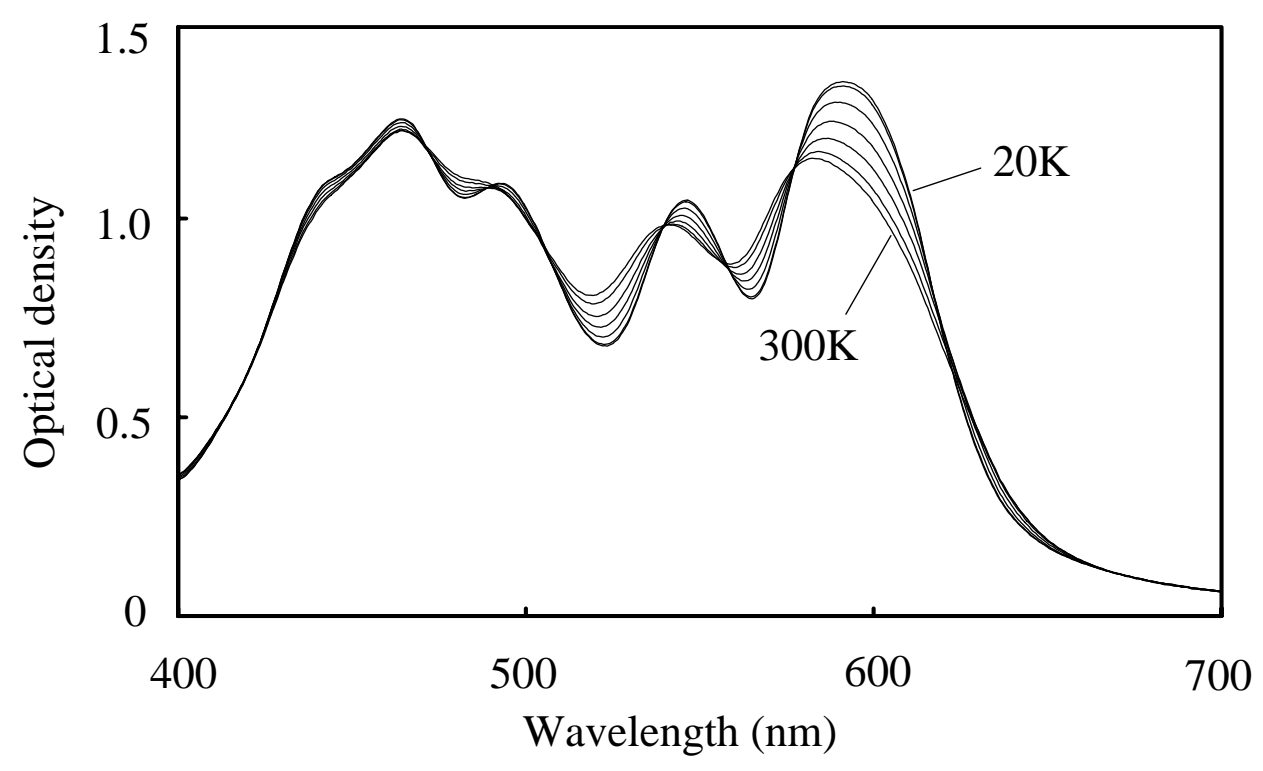

Fig. 6 
(a)

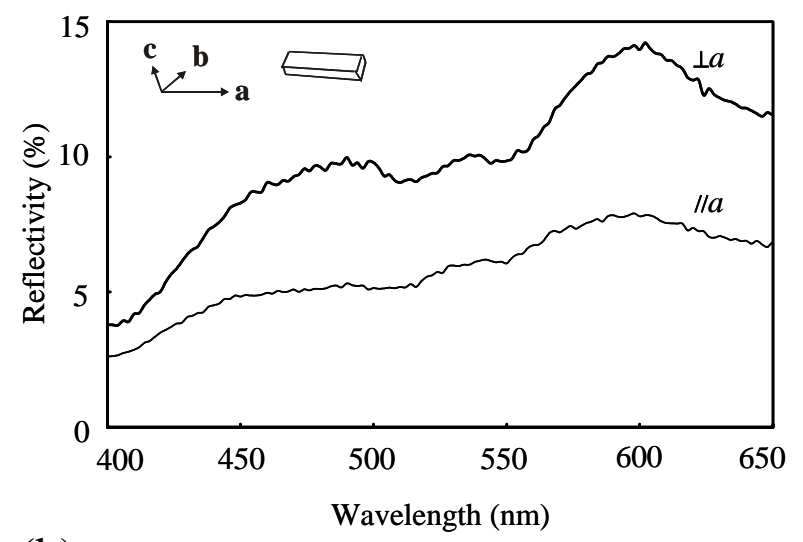

(b)

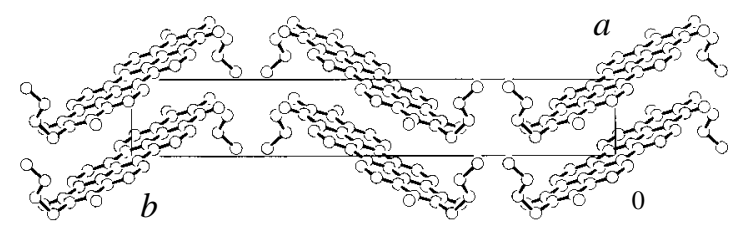

Fig. 7

(a)

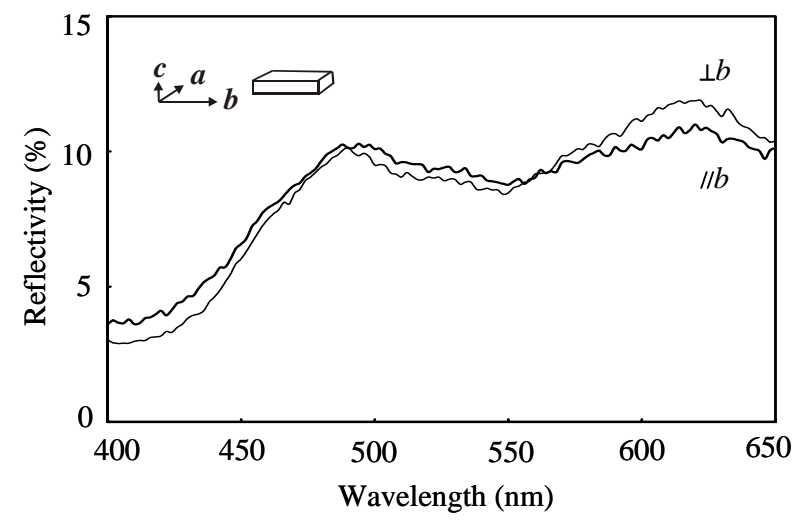

(b)

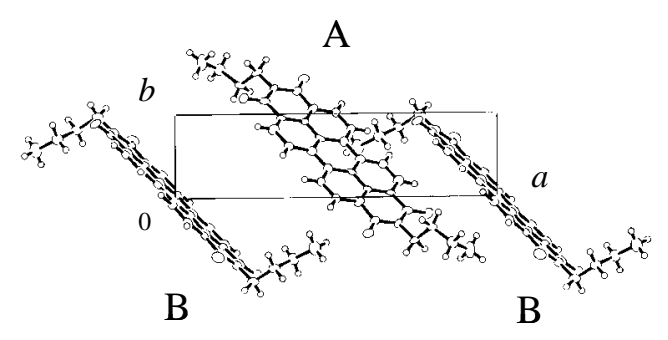

Fig. 8 